\title{
Automatic Self-Calibration of Body Worn Triaxial-Accelerometers for Application in Healthcare
}

\author{
Matthias Gietzelt, Klaus-Hendrik Wolf, Michael Marschollek, Reinhold Haux
}

\begin{abstract}
In healthcare acceleration sensors have various applications in monitoring movements for prevention, diagnostics, therapy and rehabilitation. Unfortunately they react sensitive against changes of environmental conditions and therefore it is necessary to calibrate them from time to time. This paper introduces a technique for automatic self-calibration of body worn triaxial-accelerometers that do neither need any user interaction nor a special calibration procedure. The data are calibrated automatically during operation of the sensor. The method does not require any knowledge about the actual orientation of the sensor and uses the activity of the subject to calibrate, because the activity is the region of interest for evaluation. The error of the automatic self-calibration will be as small as the error of common techniques for calibration that need an additional procedure, whereby an important error source will be excluded. The resulting algorithm is so small and compact, that it can even be transferred onto a sensor system and executed by its microprocessor.
\end{abstract}

Index Terms-Acceleration, Calibration, Pattern recognition, Wearable sensor

\section{INTRODUCTION}

UE to technological progress in integrated circuits sensors become more affordable, whereby the sample rate D ases. Therefore, the area of application extends rapidly. cially in healthcare are a lot of possible applications e.g. monitoring patients during surgery [1], in telemedicine [2] or in home care programs [3]. Within this context, acceleration sensors become more interesting in supervising the motion sequences of patients, because accelerometers are very small and deployable for miscellaneous purposes.

Acceleration data contain an immense amount of coded information. The measured data do not only contain information about the relative direction of gravity but also about velocity and the covered distance over a certain time. Especially in application with subjects it is easily possible to acquire and to interpret data about activities of daily living (e.g. sitting, lying, standing, going and climbing stairs) [4]. On the one hand high risk patients of cardiovascular diseases

Manuscript submitted September 14th, 2007.

M. Gietzelt is graduate student at the Institute of Medical Informatics of the Technical University Carolo-Wilhelmina, Braunschweig, Germany (email: m.gietzelt@tu-bs.de). could be monitored, e.g. by a step counter to give them an overview of their exercises [5]. On the other hand patients with pathologic gait could be trained in the physical therapy or at home [6].

Yet sensors need to be calibrated to provide reliable data. In the process, resulting expenses depend on the physical, chemical or biological property measured. Calibration poses a lot of problems: on the one hand a calibration process generally has to be done by an experienced specialist. On the other hand a sensor can not serve its actual purpose during the calibration. Thus a sensor has to be removed from the appropriate installation and checked according to its application.

Previous calibration algorithms for accelerometers, as introduced in [7-10], use rotational measurement. By rotational measurement each axis is lined up once in direction and once in opposite direction of gravity as precisely as possible (figure IV shows a possible result). For example not exactly leveled or uneven surfaces or changes of environmental conditions will cause an appreciable error.

In this paper an algorithm is presented which is not based on rotational measurement and which is capable of calibration during operation without knowledge of the sensor's properties. This is done by probabilistic analysis of the data. Thus, the sensor neither needs to be removed nor to be adjusted in a special calibration procedure. If no special calibration procedure is needed, an important error source will be excluded.

\section{OBJECTIVES}

The aims of this paper are:

1. to specify an automatic self-calibration algorithm for triaxial accelerometers

2. to indicate its advantage compared to calibration by rotational measurement

\section{METHODS}

\section{A. Automatic self-calibration}

Automatic self-calibration features autonomous calibration of the sensor and normalization into the unit $g=m / s^{2}$ without any configuration and input of characteristics. This is made possible by probabilistic techniques and an attentive 
observation of the behavior of acceleration data. Only a few steps that are easy to calculate are necessary for this technique. Thereby, it is assumed that the accelerometer is worn on the torso, because a wearing position close to the barycenter of the body naturally causes comparably little noise. Therefore, let $a$ $=\left(a_{1}, a_{2}, a_{3}\right)^{t}$ be the acceleration vector.

1. Determination: The three mean values $m_{1}, m_{2}$ and $m_{3}$ of the three-dimensional acceleration vectors are calculated during the times of the data, where the subject moves. This significantly reduces the error of the algorithm. The decision for the ,active" regions was obvious, because in general the measured data should be used to measure the moving behavior of the subject and therefore it is of interest for evaluation. For the computation of the mean the arithmetic mean can be used. In the cases where fast convergence is important (e.g. onlinecalibration, like calibrating during operation) the median can be used.

It emerges $a^{\prime}:=a-\left(m_{1}, m_{2}, m_{3}\right)^{t}$.

2. Prescaling: Determination of the average length of vector $a^{\prime}$ by

$$
\overline{\left\|a^{\prime}\right\|}=\frac{1}{n} \sum_{i=1}^{n} \sqrt{a_{1}^{\prime 2}+a_{2}^{\prime 2}+a_{3}^{\prime 2}}
$$

and computation of

$$
a^{\prime \prime}:=\frac{a^{\prime}}{2 \cdot \overline{\left\|a^{\prime}\right\|}}
$$

$a^{\prime}$ has to be divided by its average length to normalize the vector and to eliminate existing range information. The factor $1 / 2$ was figured out by reviewing test-sets of about 150 minutes length. This improved the results significantly.

3. Relative location: The relative location of the means to each other has been disturbed apparently by the first step. To involve the location relation of the vectors again, an offsetvector has to be calculated. Therefore, without loss of generalization let be $m_{1}>m_{2}>m_{3}$. The term in the middle $m_{2}$ is used as a fixed point and is set to zero ${ }^{1}$. The other two components, that are $a_{1}^{\prime \prime}$ and $a_{3}^{\prime \prime}$, can be displaced according to the ratio of their means (see figure I).

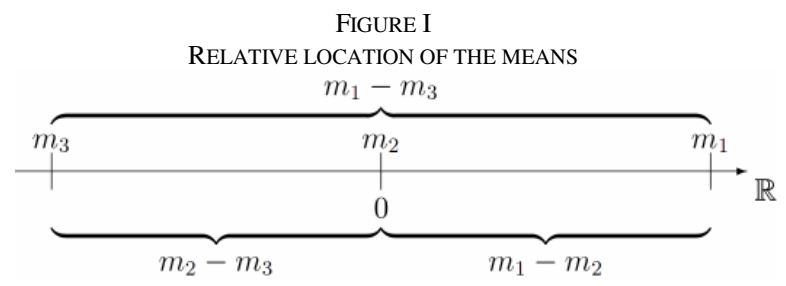

After this observation it is possible to construct the offsetvector by the ratio of the means to each other. In the case

\footnotetext{
${ }^{1}$ the value $a_{2}^{\prime \prime}$ will represent the transversal axis, because of little motion expected compared to the other axes. The "no-displacement" should indicate, that the average expected activity in this axis approaches zero.
}

above emerges:

$$
\text { offset }=\left(\frac{m_{1}-m_{2}}{m_{1}-m_{3}}, 0,-\frac{m_{2}-m_{3}}{m_{1}-m_{3}}\right)^{t}
$$

Generally framed: if for any $x, y, z \in\{1,2,3\}$ the order is $m_{x}>m_{y}>m_{z}$, whereas $x, y, z$ are pairwise disjointed, then is

$$
\begin{aligned}
& \text { offset }_{x}=\frac{m_{x}-m_{y}}{m_{x}-m_{z}} \\
& \text { offset }_{y}=0 \\
& \text { offset }_{z}=-\frac{m_{y}-m_{z}}{m_{x}-m_{z}}
\end{aligned}
$$

Therefore finally $a^{\prime \prime \prime}:=a^{\prime \prime}+$ offset .

\section{B. Activity detector}

The quality of the results can be improved, if the mean is only calculated based on the active regions of the data set, like in the first step of the automatic self-calibration. The difficulty is that the automatic self-calibration requires recognized active phases. Therefore an algorithm is needed, that will work independently on calibrated data.

The idea is to determine occurring deviation $m_{i}$ to the mean $\bar{m}_{i}$ in an observation window. The signal level of akinetic patterns changes only marginally and $m_{i}$ can be matched to the average deviation $m$ of the data set. In case of $m_{i} \ll m$ it might be a passive action. The following equations reflect the concept:

$$
\begin{aligned}
& m_{i}(\text { axis })=\int_{x_{i}}^{y_{i}} \mid a_{\text {axis }}(t)-\bar{m}_{i}(\text { axis }) \mid d t \\
& \bar{m}_{i}(\text { axis })=\frac{1}{y_{i}-x_{i}} \sum_{k=x_{i}}^{y_{i}} a_{\text {axis }}(k)
\end{aligned}
$$

whereas the observation window $i$ spans the range $\left[x_{i}, y_{i}\right] . a_{z}$ represents the $z$-coordinate of the appropriate acceleration signal $a$, whereas $a_{z}(j)$ refers to the signal level at point $j \in \mathbb{N}$. In a consequence of equation (2) an axis independent equation for $m_{i}$ is:

$$
m_{i}=\frac{1}{3} \cdot \sum_{a x i s \in\{x, y, z\}} m_{i}(\text { axis })
$$

These values are compared to:

$$
m=\frac{1}{3} \cdot \sum_{a x i s \in\{x, y, z\}} \sum_{i} m_{i}(\text { axis })
$$

for all $i$.

From $m_{i} \ll m$ follows, that the quotient can be estimated through a threshold value $s$ :

$$
\frac{m_{i}}{m}<s
$$

If falling below the threshold value $s$ it is a passive activity. Such an assumption as a quotient is reasonable, because the included range information of $m_{i}$ and $m$ are eliminated. This means that non-calibrated data can be handled. 
Example: when 8-bit encrypted data in the range $[0,255]$ are fed into the algorithm, the same threshold value $s$ can be used for data which are defined in a range $[-4,4]$.

\section{Results}

To review the quality of the self-calibration a showcase data set was recorded. With this data set it is possible to compare the self-calibration method presented in this paper to the calibration by rotational measurement. The quality of the rotational measurement can be determined by recording a calibration data set, where each axis is lined up once in direction and once in opposite direction of gravity, so that each axis is exposed to $1 g$ and $-1 g$.

The showcase data set consists of acceleration data of about an hour emphasized relaxed actions. The data set has been recorded with a sensor board from Freescale „ZSTAR Triaxial Accelerometer demo board" with the designation RD3152MMA7260Q [11]. In this process the data were recorded with $20 \mathrm{fps}$ in an 8-bit resolution. The maximum acceleration was $4 g$.

FIGURE II

A DATASET WITH VARIOUS MOTION PROCESSES

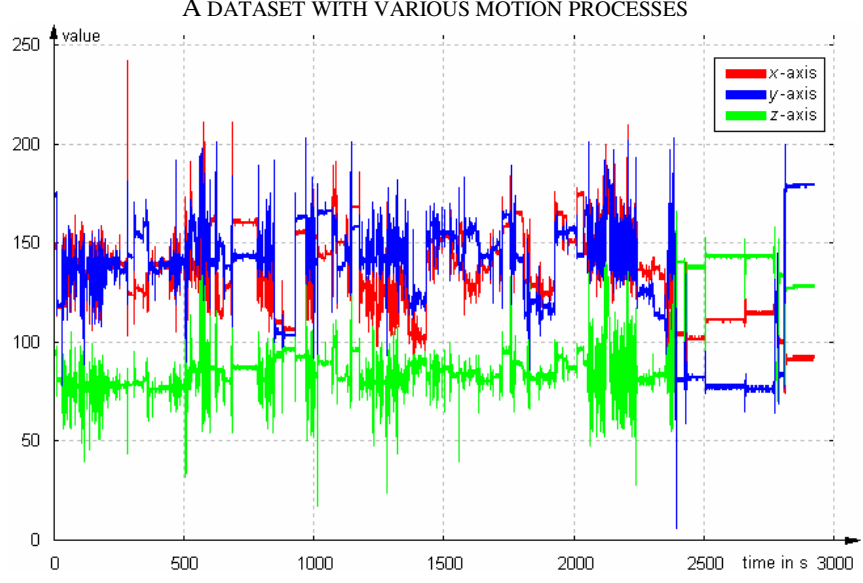

The table I decodes the actions of the data set of figure II with their corresponding durations.

TABLE I

THE DECODED ACTIONS OF THE DATA SET OF FIGURE II

\begin{tabular}{cccc}
\hline \hline begin & Stop & duration in $\mathrm{s}$ & action \\
\hline 0 & 200 & 200 & stand \\
200 & 500 & 300 & sit \\
500 & 600 & 100 & go \\
600 & 1200 & 600 & sit \\
1200 & 1400 & 200 & stand \\
1400 & 2050 & 650 & sit \\
2050 & 2250 & 200 & go \\
2250 & 2450 & 200 & sit \\
2450 & 2950 & 500 & lie down \\
\hline \hline
\end{tabular}

If the automatic self-calibration is done as presented in this paper, then an absolute error regarding rotational measurement occurs like shown in figure III. The revealed error is remarkably small and constant. If the computed error of the test data set is added up, then the sum of the absolute error will be $2727.32 \mathrm{~g}$ on 29226 triaxial values. The automatic selfcalibration, regarding to the rotational measurement that can be the only basis for the comparison here, has an average error of

$$
\begin{gathered}
\frac{\text { sum of the error in } g}{\text { number of values }}=\frac{2727.32 \mathrm{~g}}{29226}=0.093 \mathrm{~g} . \\
\text { FIGURE III }
\end{gathered}
$$

THE ABSOLUTE ERROR OF THE AUTOMATIC SELF-CALIBRATION REGARDING ROTATIONAL MEASUREMENT

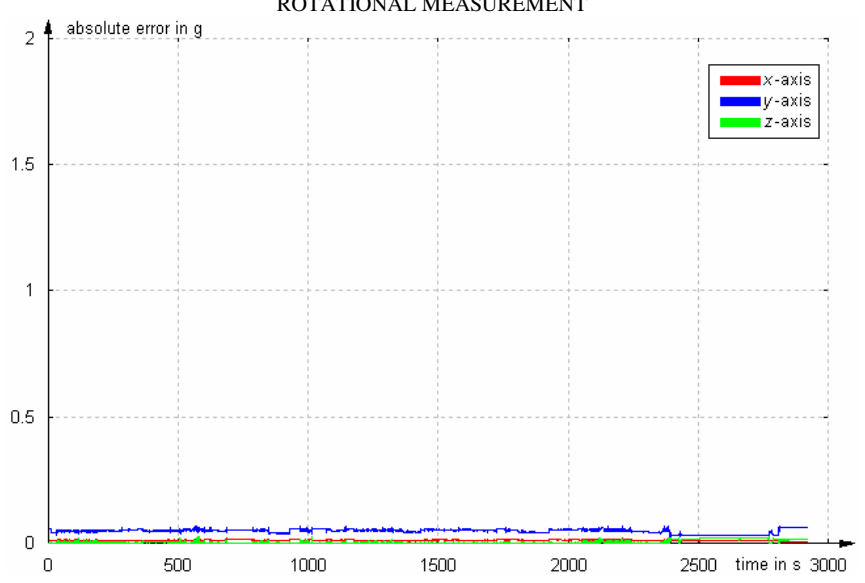

But how does the result respectively the error occurring in rotational measurement look like? Figure IV demonstrates a data set occurring in rotational measurement. Hence, six measurements were performed for about 100 seconds, to gain a representative result.

FIGURE IV

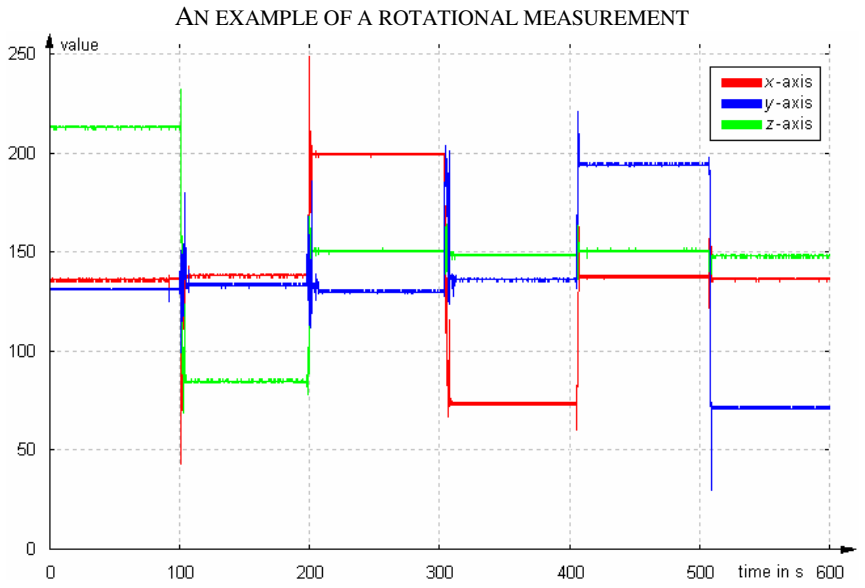

The error that occurred during rotational measurement can be shown in a diagram. Figure $\mathrm{V}$ shows the absolute error regarding the real values, because it is not possible to align the sensor accurately to gravity. The calculated sum of the errors of this data set regarding the real values is $392.27 \mathrm{~g}$ on 7600 three dimensional values. Of course the peaks during the six times of rotation of the sensor were ignored.

Now it is easy to determine the average deviation of the rotational measurement in regard to the real values:

$$
\frac{\text { sum of the error in } g}{\text { number of values }}=\frac{392.27 \mathrm{~g}}{7600}=0.052 \mathrm{~g} \text {. }
$$


FIGURE V

THE ABSOLUTE ERROR OCCURRED DURING ROTATIONAL MEASUREMENT

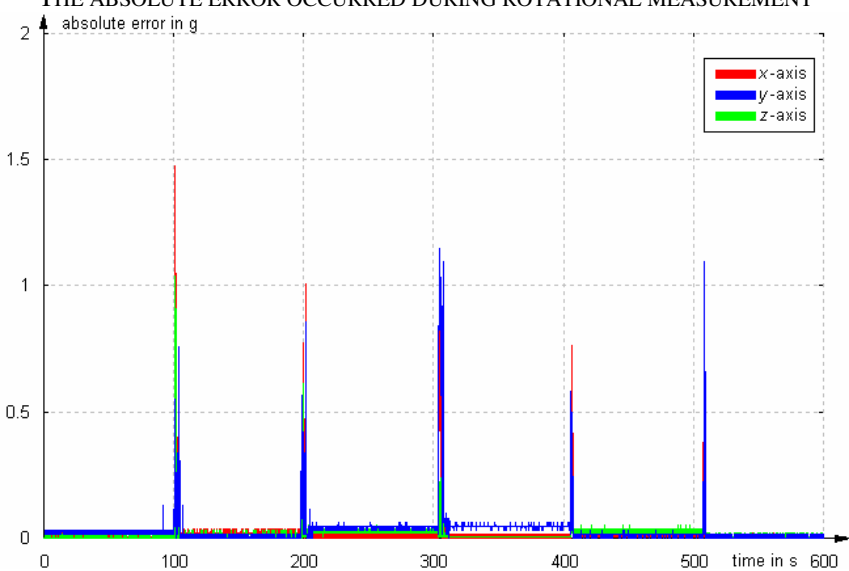

If the worst case is assumed and both errors are added, then the maximal uncertainty is $0.052 g+0.093 g=0.145 g$ regarding the real values. However, that the error is much below this can be proven easily: During a lying-down phase a significant small error occurs (see figure III). In this case the error of the automatic self-calibration regarding the real values can be determined and its average is $0.009 \mathrm{~g}$.

Sensors react sensitive against changes of environmental conditions. Especially the temperature can influence the results badly. Figure VI shows the data measured by a shadowy lying sensor during a sunny summer day. The sensor was exposed to a changing temperature and as a result the measured values increased.

FIGURE VI

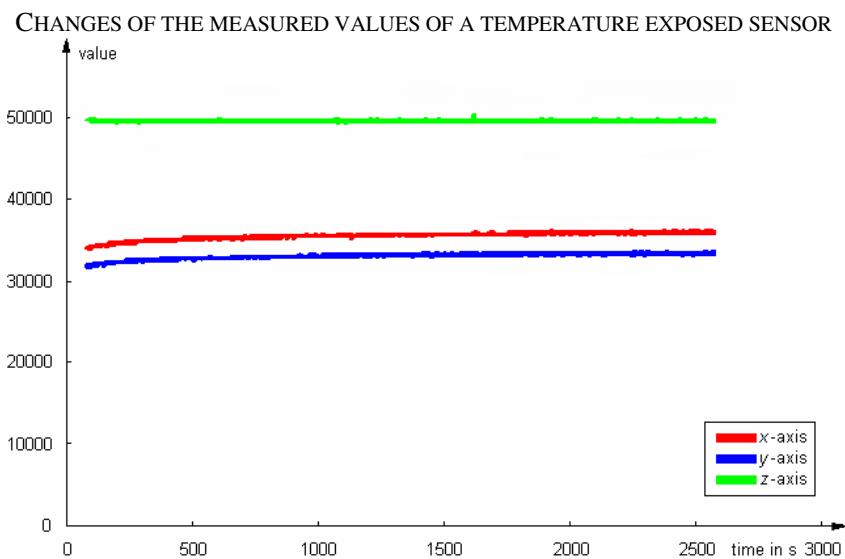

V. CONCLUSION

In this section the objectives are examined.

\section{A. Specify an automatic self-calibration algorithm (aim 1)}

The error of the automatic self-calibration (see figure III) is very small compared to conventional techniques for calibration of acceleration sensor data. Therefore, this aim has been apparently achieved. Unfortunately the used showcase did not meet demands. The results of the showcase have to be verified by public accessible case studies or data from prolective studies.

\section{B. Indicate its advantage compared to previous calibration} algorithms (aim 2)

The revealed errors indicate that the automatic selfcalibration has reached a sophisticated state and can compete against the error-prone rotational measurement if enough acceleration data for the calibration algorithm are provided. Even small variations on the measurement setup (i.e. not exactly leveled surfaces, uneven surfaces, not exactly aligned sensors regarding gravity, changes of environmental conditions etc.) can cause markedly errors. Overall it means that the automatic self-calibration eliminates a critical error source: the manual calibration. The error of rotational measurement regarding the real values is marginal smaller than the error of the automatic self-calibration regarding the rotational measurement.

Especially the adaptability of the self-calibration to changing environmental conditions (e.g. temperature, pressure etc.) through the continuous calculation of the mean has an obvious advantage compared to the rotational measurement. The occurring error of common calibration techniques will only be compensated by a frequent rotational measurement.

This paper showed that the self-calibration algorithm neither requires any maintenance nor a special calibration procedure. Body mounted sensors can be automatically calibrated during operation. Estimates suggest that the self-calibration algorithm consists only of simple operations and is so small and compact that it can be easily transferred right to a sensor system and executed by its microprocessor.

\section{REFERENCES}

[1] MO. Schurr, S. Schostek, CN. Ho, F. Rieber, A. Menciassi. Microtechnologies in medicine: An overview. Minimally Invasive Therapy and Allied Technologies 2007; Vol. 16(2), $76-86$

[2] OJ. Bott, M. Marschollek, KH. Wolf, R. Haux. Towards New Scopes: Sensor Enhanced Regional Health Information Systems - Part 1: Architectural Challenges. Methods Inf Med. 2007; 46(4): 476-83

[3] S. Shepperd, S. Iliffe. Hospital at home versus in-patient hospital care. Cochrane Database Syst Rev. 2005(3): CD000356.

[4] MJ. Mathie. Monitoring and Interpreting Human Movement Patterns Using a Triaxial Accelerometer. PhD thesis, University of New South Wales, Sydney, Australia, 2003

[5] W. Chen, D. Wei, M. Cohen, S. Ding, S. Tokinoya, N. Takeda. Development of a Scalable Healthcare Monitoring Platform. CIT 2004; 912- 5

[6] LZ. Rubenstein, PR. Trueblood. Gait and Balance Assessment in Older Persons. Annals of Long-Term Care: Clinical Care and Aging 2004; 12[2]:39-45

[7] H. Weinberg. Using the adxl202 duty cycle output. iMEMS Technologies/Applications AN-604, Analog Devices, 1998

[8] JC. Lötters, J. Schipper, PH. Veltink, W. Olthuis, P. Bergveld. Procedure for in-use calibration of triaxial accelerometers in medical applications. Sensors and actuators. A, Physical 1998; Vol. 68: 221-8

[9] P. Lukowicz, H. Junker, G. Troster. Automatic calibration of body worn acceleration sensors. In Proceedings of the second international Pervasive Computing conference, Vienna, Austria, 2004

[10] S. Scapellato, F. Cavallo, C. Martelloni, AM. Sabatini. In-use calibration of body-mounted gyroscopes for applications in gait analysis. Sensors and actuators. A, Physical 2005; Vol. 123-124: 418-22

[11] Freescale Semiconductor, ZSTAR. Wireless Sensing Triple-Axis Reference Design (ZSTAR). Document Number: ZSTARBOARDFS. [Online]. 2007 [cited Aug, 25th 2007]; Available: http://www.freescale.com/files/sensors/doc/fact_sheet/ZSTARBOARDF S.pdf 